BINP 95-22

UQAM-PHE/95-03

March 1995

\title{
Electric dipole moment of neutron in the Kobayashi-Maskawa model with four generations of quarks.
}

\author{
C. Hamzaoui円 \\ Département de Physique, Université du Québec à Montréal, \\ Case Postale 8888, Succ. Centre-Ville, Montréal, Québec, Canada, H3C 3P8.
}

and

M.E. Pospelovt

Budker Institute of Nuclear Physics, 630090 Novosibirsk, Russia

\begin{abstract}
We show that the existence of a possible fourth heavy generation of quarks gives rise to a significant enhancement to the neutron electric dipole moment in comparison with the Standard Model prediction. The smaller degree of suppression in this case is linked to the presence of the operators of dimension $\leq 6$ which enter into the effective Lagrangian with coefficients proportional to the square of the top quark mass. Numerically, the enhancement is mainly associated with chromoelectric dipole moment of the s quark which appears at three loop level, of the order $\alpha_{s} \alpha_{w}^{2} m_{s} m_{t}^{2} / m_{w}^{4}$ from the CP-odd combination of mixing angles between second, third and fourth generations. Its value is calculated explicitly in the limit of large masses of the fourth generation of quarks. The corresponding contribution to the electric dipole moment of the neutron is $5 \cdot 10^{-30} \mathrm{e} \cdot \mathrm{cm}$ in the most optimistic scenarios about the values of the Kobayashi-Maskawa matrix elements. The additive renormalization of $\theta$-term in this model is estimated as $10^{-13}$.
\end{abstract}

\footnotetext{
${ }^{1}$ E-mail: hamzaoui@mercure.phys.uqam.ca

${ }^{2}$ E-mail: pospelov@inp.nsk.su
} 


\section{Introduction}

The Kobayashi-Maskawa (KM) model looks now as the most natural description of CPviolation. It describes properly $\mathrm{CP}$-odd phenomena in the decays of neutral $K$-mesons and predicts extremely tiny CP-odd effects in the flavour-conserving processes. The current experimental limit on the electric dipole moment of neutron (EDM) [1],

$$
d_{N} / e<10^{-25} \mathrm{~cm}
$$

exceeds the realistic Standard Model prediction for this quantity by seven orders of magnitude. However, this gap between theory and experiment is of great use for limiting a new CP-violating physics beyond the Standard Model. The purpose of this work is to consider the electric dipole moment of neutron in the model with an additional heavy generation of quarks preserving the same KM origin of CP-violation.

The reason for introducing an extra heavy generation of quarks into physics comes from a relatively large mixing in $B-\bar{B}$ meson system [2]. Its existence is, of course, questionable. However, the set of constraints on mixing angles and unknown heavy masses can be derived from the low energy phenomenological data. The analysis [2] shows that the existence of additional heavy quarks with masses not lighter than $m_{t}$ is not excluded. We would use the conclusions developed in the work [2] for possible values of KM matrix elements in terms of the Wolfenstein parameter $\lambda=\left|V_{u s}\right|=0.22$.

Let us denote the fourth generation flavours as ( $\mathrm{h} \mathrm{g}$ ). Then the best scenario about a large mixing between the third and fourth generations consistent with current experimental data is given by:

$$
\left|V_{h b}\right| \sim\left|V_{t g}\right| \sim \mathcal{O}(\lambda)
$$

This scenario is quite natural if we assume the masses of $h$ and g quarks lying below the perturbative unitarity limit of $500 \mathrm{GeV}$. Using the unitarity conditions for the KM matrix and already known values of matrix elements we deduce the following:

$$
\left|V_{c g}\right| \sim\left|V_{h s}\right| \sim \mathcal{O}\left(\lambda^{2}\right) ;\left|V_{h d}\right| \sim\left|V_{u g}\right| \sim \mathcal{O}\left(\lambda^{3}\right)
$$

The enlarged KM matrix of this model possesses 9 independent parameters which include six mixing angles and three CP-violating phases. To avoid the uncertainties of reparametrization, we would describe all CP-odd flavour-diagonal amplitudes in terms of imaginary part of three independent quartic combinations of KM matrix elements:

$$
\begin{aligned}
\operatorname{Im}\left(V_{t d}^{*} V_{t b} V_{c b}^{*} V_{c d}\right) \sim \mathcal{O}\left(\lambda^{7}\right) ; & \operatorname{Im}\left(V_{t d}^{*} V_{t b} V_{h b}^{*} V_{h d}\right) \sim \mathcal{O}\left(\lambda^{7}\right) \\
\operatorname{Im}\left(V_{t s}^{*} V_{t b} V_{h b}^{*} V_{h s}\right) & \sim \mathcal{O}\left(\lambda^{5}\right)
\end{aligned}
$$

All other rephasing invariants could be reexpressed using these three combinations and moduli of KM matrix elements only. 


\section{Standard Model prediction}

Let us start from the Standard Model (SM) prediction for the electric dipole moment (EDM) of neutron. The violation of the CP-symmetry in the Standard Model originates from the sole complex phase in the KM matrix. To lowest, quadratic order in the weak interaction all CP-odd flavour-conserving amplitudes turn to zero trivially. The point is that in this approximation those amplitudes depend only on the moduli squared of elements of the KM matrix, so the result cannot contain the CP-violating phase. In the next, quartic order in semi-weak coupling constant $g_{w}$, the expression for EDMs of quarks vanishes at two-loop approximation without hard gluon radiative correction taken into account [3]. It can be shown, however, that the inclusion of one hard gluon loop prevents EDMs from identical cancellation.

The values of EDMs to one gluon loop accuracy was calculated first by Khriplovich [4]. We quote here his result:

$$
d_{d}=e \tilde{\delta} \frac{\alpha_{s} G_{F}^{2} m_{c}^{2} m_{d}}{108 \pi^{5}} \log \frac{m_{t}^{2}}{m_{b}^{2}} \log ^{2} \frac{m_{b}^{2}}{m_{c}^{2}} \simeq e \cdot 2 \cdot 10^{-34} \mathrm{~cm}
$$

where $G_{F}=\sqrt{2} g_{w}^{2} /\left(8 M^{2}\right)$ is the Fermi constant and $m_{i}$ is the mass of i-flavoured quark. $\tilde{\delta}$ denotes here the only possible CP-odd invariant of 3 by $3 \mathrm{KM}$ matrix. The analogous result for $d_{u}$ is much smaller being proportional to $m_{s}^{2}$.

Formula (5) was obtained in the limit of the effective four-fermion contact interaction which is valid when all quark masses and characteristic loop momenta are much smaller than masses of $S U(2)$ gauge bosons. This is not exactly true in general because the topquark does not satisfy this demand. However, we could use the expression (5) as a good estimate for EDM of d-quark in the Standard Model. The reason being is that $m_{t}$ enters to (5) under logarithm only and may be replaced by $m_{w}$.

The EDM of neutron in Standard Model, however, is much larger than EDMs of its constituents due to the so called "long distance" effects [5, 6, 7]. The most reliable estimates according the rules of chiral perturbation theory predicts the EDM of neutron at the level $d_{N} \simeq 2 \cdot 10^{-32} \mathrm{e} \cdot \mathrm{cm}$. The two orders of magnitude enhancement here is basically due to a smaller number of closed loops and bigger factor connected with strong interactions.

Our basic idea is to extend this consideration to the case of KM model with an additional heavy generation of fermions. The reason is that instead of one CP-violating phase, the enlarged variant of this model possesses three CP-odd parameters and additional flavour's combinations in the amplitudes. It may cause the contributions to EDMs being proportional to the square of the mass of the top-quark. As a result, short distance contributions to EDMs of quarks would be suppressed not by $G_{F}^{2} m_{c}^{2}$ but rather by $\alpha_{w} G_{F}$.

In general, the problem of EDM calculation should be divided into two independent parts. First, we construct a low energy effective Lagrangian in terms of $\mathrm{u}$, d, s quarks, gluons and external electromagnetic field. Then, we recalculate this Lagrangian to the EDM of neutron using all available methods for doing low energy hadronic physics. The second part is independent from the concrete model of CP-violation at high energies. It is clear that in our case the possible enhancement should be associated with effective 
operators of low dimension, not bigger than 6. It is easy to identify all these possible structures. Integrating out c, b, t, g, h quarks and $\mathrm{SU}(2)$ gauge fields, we construct a low energy effective Lagrangian in the form:

$\mathcal{L}_{e f f}(x)=\theta \frac{\alpha_{s}}{8 \pi} G_{\mu \nu}^{a} \tilde{G}_{\mu \nu}^{a}+i \frac{c_{W}}{6} g_{s}^{3} f^{a b c} \tilde{G}_{\alpha \beta}^{a} G_{\beta \mu}^{b} G_{\mu \alpha}^{c}+\sum_{i} \frac{\tilde{d}_{i}}{2} g_{s} \bar{q}_{i} t^{a}\left(G^{a} \sigma\right) \gamma_{5} q_{i}+\sum_{i} \frac{d_{i}}{2} e \bar{q}_{i}(F \sigma) \gamma_{5} q_{i}$,

where $(F \sigma)$ denotes $F_{\mu \nu} \gamma_{\mu} \gamma_{\nu}$ and the summation is held over light flavours: $i=u, d, s$. The first term in (6) represents the induced $\theta$-term, perturbative contribution to the total $\theta$-term of the theory. The next operator of dimension 6 was introduced originally by Weinberg [8]; the SM value of corresponding coefficient $c_{W}$ was calculated in [9]. Other terms in (6) with dim $=5$ are the operators of EDMs and chromoelectric dipole moments (CEDM). The dimension 5 of these operators in some sense is fictitious because of a chirality flip making coefficients $\tilde{d}_{i}$ to be proportional to light masses $m_{i}$. Therefore, both $d_{i}$ and $\tilde{d}_{i}$ are suppressed by at least two powers of heavy mass corresponding to the weak interaction scale. Other CP-odd operators of dimensions higher than 6 are unimportant in our consideration because they are suppressed by additional powers of heavy masses.

Some comments should be made at this point. We do not add to (6) CP-odd mass operators of quarks $i \bar{q}_{i} \gamma_{5} q_{i}$ because they could be incorporated to the $\theta$-term by mean of the chiral rotation. Other flavour conserving $\mathrm{CP}$-odd operators of dim $=6$ built from four quark fields are suppressed in comparison with CEDMs. The last fact refers to our concrete model of CP-violation. Because of the $\mathrm{V}$ - A character of the theory, effective operators are originally formulated in terms of left handed fields. In its turn, CP-violation may only arise through the chirality flip which gives extra powers of light mass and affects the suppression of four quark operators. This last remark is connected with the so called "axial polyp operators" introduced originally in [10]:

$$
c_{P} \bar{q}_{i} \gamma_{\mu}\left\{G_{\mu \nu}, \vec{D}_{\nu}\right\} \gamma_{5} q_{i}
$$

This operator is identical to CEDM one if we assume the equations of motion to be preserved:

$$
i \gamma_{\mu} D_{\mu} q_{i}=m_{i} q_{i}
$$

Further analysis is recalled to single out leading operators in (6) and find corresponding coefficients.

\section{$3 \quad$ Flavour's structure of $\mathcal{L}_{e f f}$}

We start investigating the coefficients in (6) by determining the flavour's arrangement along a fermion line. Let us denote by $\mathrm{f}$ the Green function of f-flavoured fermion. Then a CP-odd amplitude for fermionic operators in quartic order in semi-weak constant could be written in the following form:

$$
\sum_{j, k, l} i \operatorname{Im}\left(V_{j f}^{*} V_{j k} V_{l k}^{*} V_{l f}\right) f j k l f .
$$


For pure gluonic operators the corresponding structure looks as:

$$
\sum_{f, j, k, l} i \operatorname{Im}\left(V_{j f}^{*} V_{j k} V_{l k}^{*} V_{l f}\right) f j k l,
$$

where the cyclic permutation of the kind $f j k l=l f j k=k l f j=j k l f$ is allowed.

In three generation formulation of KM model one has the only source of CP violation which fixes unique flavour structures for both type of operators [四. In the four family case, the number of CP violating phases is three. Their concrete choice could be done in a different ways complicating our analysis. However, it does not affect the main property responsible for the cancellation of EDMs at two loops. It is easy to see that independently on the number of families the expression (9) is antisymmetric under the interchange of flavours $\mathrm{j}$ and $\mathrm{l}$ :

$$
\sum_{j, k, l} i \operatorname{Im}\left(V_{j f}^{*} V_{j k} V_{l k}^{*} V_{l f}\right) f j k l f=\frac{1}{2} \sum_{j, k, l} i \operatorname{Im}\left(V_{j f}^{*} V_{j k} V_{l k}^{*} V_{l f}\right) f(j k l-l k j) f .
$$

This antisymmetry is sufficient to set to zero both EDM and CEDM of quark to two-loop approximation [3].

It is useful to classify a variety of CP-odd amplitudes by mean of dynamical arguments. The enhancement of the short distance contributions to the $\mathcal{L}_{\text {eff }}$ which we expect to get is closely related to the fact that all characteristic loop momenta are comparable with the weak interaction scale. Therefore, inside the loops, we are legitimate to put all quark masses to zero except $m_{t}, m_{g}$ and $m_{h}$. In other words, inside the loops, we are able to identify propagators of light quarks:

$$
c=u \equiv U ; \quad d=s=b \equiv D .
$$

It should be mentioned here that $\mathrm{c}$ and b quarks play a twofold role. They are considered as heavy quarks at normal hadronic scale and almost massless inside loops, at the scale of weak interactions. This requirement immediately leads to the vanishing of pure gluonic operators as well as EDM and CEDM of u-quark. Indeed, applying the unitarity condition for the KM matrix

$$
V_{d u}^{*} V_{d k}+V_{s u}^{*} V_{s k}+V_{b u}^{*} V_{b k}=\delta_{u k}-V_{g u}^{*} V_{g k}
$$

we perform the summation over $j$ and $l$ in (9) explicitly:

$$
\sum_{j, k, l} i \operatorname{Im}\left(V_{j u}^{*} V_{j k} V_{l k}^{*} V_{l u}\right) u j k l u=\sum_{k} i \operatorname{Im}\left(V_{g u}^{*} V_{g k} V_{g k}^{*} V_{g u}\right) u(D k g-g k D) u=0 .
$$

For s and d quark operators the situation is quite different. An analogous procedure for them leads to the following combination:

$$
\frac{1}{2} \sum_{k} i \operatorname{Im}\left(V_{t f}^{*} V_{t k} V_{h k}^{*} V_{h f}\right) f[t k h-h k t+U k t-t k U+h k U-U k h] f,
$$

where $f=d, s$.

Now we will concentrate on s-quark operators because its mixing with third and fourth generations is a priori bigger than that of d-quark. 
Taking the last sum over $k$ we obtain:

$\frac{i}{2} \operatorname{Im}\left(V_{t s}^{*} V_{t g} V_{h g}^{*} V_{h s}\right) s[t(g-D) h-h(g-D) t+U(g-D) t-t(g-D) U+h(g-D) U-U(g-D) h] s$.

The rephasing invariant combination of KM matrix elements in (16) to a good accuracy coincides with that responsible for $\mathrm{CP}$-odd $B_{S}^{0}$ meson mixing:

$$
\operatorname{Im}\left(V_{t s}^{*} V_{t g} V_{h g}^{*} V_{h s}\right)=-\operatorname{Im}\left(V_{t s}^{*} V_{t b} V_{h b}^{*} V_{h s}\right),
$$

and the resulting expression takes the form:

$$
\frac{i}{2} \operatorname{Im}\left(V_{t s}^{*} V_{t b} V_{h b}^{*} V_{h s}\right) s[t(b-g) h-h(b-g) t+U(b-g) t-t(b-g) U+h(b-g) U-U(b-g) h] s .
$$

It may be of the order $\lambda^{5}$ and it makes the electric and chromoelectric dipole moment of s-quark the most important between other CP-odd operators.

For the d-quark all considerations presented above are valid with the only replacement in rephasing invariants:

$$
\operatorname{Im}\left(V_{t s}^{*} V_{t b} V_{h b}^{*} V_{h s}\right) \longrightarrow \operatorname{Im}\left(V_{t d}^{*} V_{t b} V_{h b}^{*} V_{h d}\right) \sim \mathcal{O}\left(\lambda^{7}\right) .
$$

\section{EDM of neutron}

When the flavour structure is fixed, it is possible to find the relative meaning of different operators for the EDM of neutron using order of magnitude estimations for corresponding coefficients.

First we take an electric dipole moment of d-quark. Combining together all phase space factors, coupling constants and taking into account (19) we get the following estimate:

$$
d_{d} \sim \lambda^{7} \frac{\alpha_{s}}{4 \pi} \frac{\alpha_{w}}{4 \pi} \frac{1}{16 \pi^{2}} G_{F} m_{d} \frac{m_{t}^{2}}{m_{w}^{2}}
$$

In the next section, we will find that this estimate is reasonable. Now using a simplest constituent model, we obtain the corresponding contribution to the EDM of neutron at the level:

$$
d_{N} \simeq d_{d} \sim e \cdot 3 \cdot 10^{-32} \mathrm{~cm}
$$

It turns out that it does not exceed the Standard Model prediction. The enhancement in comparison with SM value of $d_{d}$ is just two orders of magnitude instead of $m_{t}^{2} / m_{c}^{2} \simeq 10^{4}$. It can be explained rather trivially. In contrast with the SM result (5), the estimate (20) does not possess any logarithmic enhancement. Another origin of this deficiency is related

to a smaller value of $\alpha_{s}$ in (20) and smaller numerical factor in comparison with that of (5).

In the same manner we estimate the chromoelectric dipole moment of s-quark:

$$
\tilde{d}_{s} \sim \operatorname{Im}\left(V_{t s}^{*} V_{t b} V_{h b}^{*} V_{h s}\right) \frac{\alpha_{s}}{4 \pi} \frac{\alpha_{w}}{4 \pi} \frac{1}{16 \pi^{2}} G_{F} m_{s} \frac{m_{t}^{2}}{m_{w}^{2}} \sim \lambda^{5} \frac{\alpha_{s}}{4 \pi} \frac{\alpha_{w}}{4 \pi} \frac{1}{16 \pi^{2}} G_{F} m_{s} \frac{m_{t}^{2}}{m_{w}^{2}} .
$$


The renormalization to hadronic scale does not seriously change this estimate. The recalculation of the contribution of this quantity to the EDM of neutron is a separate problem. Here we use the result of Khatsimovsky, Khriplovich and Zhitnitsky [1] which does not exhibit any additional suppression of the contribution to NEDM from this operator:

$$
d_{N} \simeq-\frac{1}{2} \tilde{d}_{s} \sim e \cdot 5 \cdot 10^{-30} \mathrm{~cm}
$$

This estimate shows that the four-generation formulation of the KM model in the most optimistic scenario about possible values of $\mathrm{CP}$-odd rephasing invariants leads to the electric dipole moment of neutron two orders of magnitude bigger than its SM value. The electric dipole moment of s-quark appears to be of the same order of magnitude as $\tilde{d}_{s}$. However, its contribution to NEDM seems to be suppressed in comparison with that of CEDM operator [11.

It is useful also to estimate values of other terms in $\mathcal{L}_{\text {eff }}$. Both the induced $\theta$-term and Weinberg operator turn out to be suppressed by the ratio $m_{b}^{2} / m_{w}^{2}$. It means also that the characteristic loop momenta could range widely, from $m_{b}$ to electroweak scale. Therefore, it is quite possible that these contributions would match also a large logarithmic factor so the total suppression would be of order $m_{b}^{2} / m_{w}^{2} \log \left(m_{w}^{2} / m_{b}^{2}\right) \sim 1 / 40$. Thus, our estimate for the induced value of $\theta$-term is:

$$
\theta \sim \lambda^{5} \frac{1}{8} \frac{\alpha_{s}}{4 \pi} \frac{\alpha_{w}}{4 \pi} \frac{m_{t}^{2} m_{b}^{2}}{m_{w}^{4}} \log \left(m_{w}^{2} / m_{b}^{2}\right) \simeq 3 \cdot 10^{-13} .
$$

This value is just three orders of magnitude smaller than the current limit on the total $\theta$-term. Its contribution to the EDM of neutron could make sense only after specifying a mechanism of CP-strong puzzle solution. It is clear that the popular elimination of $\theta$-dependence due to axions makes the EDM of neutron unfeasible for (24). There are, however, some alternative solutions for CP-strong problem which assume the $\theta$-relaxation at tree level only. This solution makes the most important the $\theta$-term contribution to the EDM of neutron.

The last operator of interest is the Weinberg operator. Its distinguishing feature is the existence without hard gluon radiative corrections taken into account [9]. However its numerical contribution to EDM in the four-family case is unlikely to exceed $10^{-33} \mathrm{e.cm}$. The additional smallness here is related to the strong suppression from the renormalization to hadronic scale.

\section{The limit of infinitely heavy $m_{h}$ and $m_{g}$}

We now check if the estimate (22) is correct enough and prove the absence of exact cancellation or additional suppression at three loop level.

The expression of interest is a three loop integral where quarks and $\mathrm{SU}(2)$ gauge bosons with comparable masses are involved. To simplify the problem, we impose on masses an artificial condition

$$
m_{h}^{2}, m_{g}^{2} \gg m_{t}^{2} \gg m_{w}^{2}
$$


which would allow to use an effective scale separation. This limit in general breaks the perturbative unitarity and forces us to take into account further electroweak loops. However, it may be used to estimate the first coefficient of perturbative series. In our case it brings some important simplification to the problem of the loop calculation. The most important contributions are then associated with longitudinal parts of W-boson's Green functions. Indeed, if the limit (25) is held, all characteristic loop momenta could range between $m_{t}$ and heaviest masses. From that scale, according to our assumption, $m_{w}$ could be regarded as a small mass and $1 / m_{w}^{2}$ in the longitudinal parts of $\mathrm{W}$-propagator makes its relative contribution to the effect to be enhanced in comparison with that of Feynman parts. Thus, it is clear that the quantity of interest, $\tilde{d}_{s}$, will be proportional to the factor $m_{t}^{2} / m_{w}^{4}$ and we omit possible contributions of order $1 / m_{w}^{2}$ and $1 / m_{t}^{2}$. In other words, it means that in t'Hooft - Feynman gauge we take into account only diagrams with charged scalar Higgses and neglect those with W-bosons. The accuracy of the assumption (25) for real massive parameters is presumably about $1 / 4$ if we keep masses of heaviest quarks around $500 \mathrm{GeV}$ and it is sufficient for our purposes.

Two different possibilities of $\mathrm{W}$-bosons attachment to the fermion line are depicted in Figs. 1a and 1b. These skeletons should be dressed by one hard gluon loop and external soft gluon leg. Our calculation of the CEDM is based upon the external field technique proposed by J. Schwinger for QED and then extended on to the QCD case by Novikov, Shifman, Vainshtein and Zakharov (see for ex. the review [12]). It can surely be applied to this problem because all loop momenta are much larger than the characteristic hadronic scale. The technique deals with the operator

$$
\left\langle x\left|P_{\mu}\right| y\right\rangle=\left\langle x\left|i D_{\mu}\right| y\right\rangle=\left(i \frac{\partial}{\partial x_{\mu}}+g_{s} \frac{\lambda^{c}}{2} A_{\mu}^{c}(x)\right) \delta^{4}(x-y)
$$

where $A_{\mu}^{c}(x)$ is the gluonic field. Then the quark propagator taken in the background gluonic field reads as:

$$
\left\langle v a c\left|T q^{a}(x) \bar{q}^{b}(y)\right| v a c\right\rangle=\left\langle x, a\left|i(\hat{P}-m)^{-1}\right| y, b\right\rangle=\left\langle x, a\left|(\hat{P}-m) \frac{i}{P^{2}+i g_{s} / 2(G \sigma)-m^{2}}\right| y, b\right\rangle,
$$

where $\hat{P} \equiv \gamma_{\mu} P_{\mu}$. The field strength originates here as a result of commutation of two $P^{\prime}$ s:

$$
\left[P_{\mu}, P_{\nu}\right]=i g_{s} G_{\mu \nu}^{a} \frac{\lambda^{a}}{2} \equiv i g_{s} G_{\mu \nu}
$$

Using this technique, it is easy to demonstrate the vanishing of CEDM at two loops. This cancellation occurs before the last integration over a momentum of outer W-boson loop. The part of the amplitude of interest, Fig. 1a, comprises mass operator between two propagators antisymmetrized in masses:

$$
\frac{\hat{P}}{\hat{P}^{2}-m_{j}^{2}} \hat{\Gamma} \frac{\hat{P}}{\hat{P}^{2}-m_{l}^{2}}-\left(m_{j} \leftrightarrow m_{l}\right),
$$

The sum over $\mathrm{i}$ and $\mathrm{j}$ is performed according the prescription (18). Here $\hat{\Gamma}$ denotes the mass operator taken in the background gluonic field. It possesses $\mathrm{V}$ - A gamma matrix structure and allows for the expansion in series of external field operators of increasing 
dimension with some invariant functions depending on $P^{2}$ as coefficients. The explicit antisymmetrization in masses in (29) leads to the following expression for this amplitude:

$$
\left(m_{j}^{2}-m_{l}^{2}\right) \frac{\hat{P}}{\left(\hat{P}^{2}-m_{j}^{2}\right)\left(\hat{P}^{2}-m_{l}^{2}\right)}\left[\hat{\Gamma},(\hat{P})^{2}\right] \frac{\hat{P}}{\left(\hat{P}^{2}-m_{j}^{2}\right)\left(\hat{P}^{2}-m_{l}^{2}\right)}
$$

The commutator in this expression could be calculated for all operators entering to $\hat{\Gamma}$. It could be shown that the result of commutation starts from operators with several powers of field strengths or field derivatives. Therefore, the two-loop amplitudes cannot induce CEDM (see Ref. 9] for details). Clearly, at three loop accuracy this commutator must be a source of hard gluon field which compensates some extra dimensions. Another point of hard gluon attachment is not fixed and all other fermion lines should be expanded up to the first order in its field. The similar procedure could be performed over diagrams of the second type.

It is clear that the result of integration may contain some power of $\log \left(m_{h}^{2} / m_{t}^{2}\right)$ or $\log \left(m_{g}^{2} / m_{t}^{2}\right)$. Naively we can regard this logarithm as a big parameter and calculate all diagrams in the "Leading Logarithm Approximation". To this approximation we believe that $\log \left(m_{h}^{2} / m_{t}^{2}\right) \ll \frac{1}{2 !} \log ^{2}\left(m_{h}^{2} / m_{t}^{2}\right) \ll \frac{1}{3 !} \log ^{3}\left(m_{h}^{2} / m_{t}^{2}\right)$. It is not true for real values of our masses. However to obtain an order of magnitude, we put all $\frac{1}{n !} \log ^{n}$ equal to 1 in the final answer. The reason for assuming this artificial condition is to simplify the set of multi-loop calculation reducing it to the consequence of factorized integrations.

It turns out that the logarithmic accuracy allows to find all relevant operators in $\hat{\Gamma}$ expansion and single out leading diagrams for the rest of amplitude. We start from the smallest distances determining the internal mass operator $\hat{\Gamma}$ for diagrams in Fig. 1a and one of mass operators in Fig. 1b. These distances are associated with the propagation of $\mathrm{g}$ and $\mathrm{h}$ quarks respectively. The $\mathrm{V}$ - A gamma-matrix structure together with gauge invariance give three possible operators:

$$
\hat{\Gamma}=\frac{1}{m_{w}^{2}}\left(c_{0} \hat{P}^{3}+c_{1} \gamma_{\nu} g_{s} D_{\mu} G_{\mu \nu}+c_{2} g_{s} \gamma_{\mu}\left\{P_{\nu}, G_{\alpha \beta}\right\} \epsilon_{\mu \nu \alpha \beta}\right) \frac{1-\gamma_{5}}{2} \equiv\left(\mathcal{O}_{0}+\mathcal{O}_{1}+\mathcal{O}_{2}\right)
$$

The choice of these operators is not unique. We could use another basis; $\left\{\hat{P}, P^{2}\right\}$, for example instead of $\hat{P}^{3}$, etc. However, the expansion (31) appears to be the most convenient. The first term in this series, $\mathcal{O}_{0}$ operator, identically vanishes being substituted into (30). For the second type of diagram this operator gives $m_{s}^{3}$ being applied to the s-quark wave function and therefore can be omitted as well. Two other operators in this series enter here with numerical coefficients $c_{1}$ and $c_{2}$ whith no momentum or mass dependencies. They could be calculated from the expansion of mass operator in $\hat{P}$ at $P^{2} \ll m^{2}$.

$$
-i \hat{\Gamma}=-\frac{g_{w}^{2}}{2 m_{w}^{2}} \int \frac{d^{4} q}{(2 \pi)^{4} q^{2}} \hat{q} \frac{1-\gamma_{5}}{2} \frac{1}{\hat{q}-m} \hat{P} \frac{1}{\hat{q}-m} \hat{P} \frac{1}{\hat{q}-m} \hat{P} \frac{1}{\hat{q}-m} \hat{q} \frac{1-\gamma_{5}}{2}
$$

We used here the W-boson's Green function in the unitary gauge:

$$
-i \frac{g_{\mu \nu}-q_{\mu} q_{\nu} / m_{w}^{2}}{q^{2}-m_{w}^{2}} \simeq i \frac{q_{\mu} q_{\nu}}{m_{w}^{2} q^{2}}
$$


It is easy to see that the logarithmic divergence of (32), converting to $\log \left(\mathrm{m}^{2} / \mathrm{p}^{2}\right)$ after applying GIM mechanism, is associated with the $\mathcal{O}_{0}$ operator only. The straightforward calculation leads to the following result:

$$
\frac{c_{1}}{m_{w}^{2}}=-\frac{\alpha_{w}}{4 \pi} \frac{1}{12} ; \quad \frac{c_{2}}{m_{w}^{2}}=-\frac{\alpha_{w}}{4 \pi} \frac{5}{48} ;
$$

We are left with two-loop expressions which could give a square of logarithm in the final answer. This means that the integration over a momentum flowing at the $t$ quark line should be performed in last turn as we integrate from small distances to large ones. The corresponding diagrams and top quark propagation are shown in Figs. 2a and 2b. The blob represents operator $\hat{\Gamma}$; dashed line here is the hard gluon propagator. It is clear that to logarithmic accuracy, the result of hard gluon loop integration may be presented as an effective vertex of $\mathrm{W}$-boson with a fermion line changing the flavour from t to s.

The position of external gluon field is not indicated in Figs. 2 and all propagators should be taken in the background gluonic field. At first look, the perturbative expansion breaks the main advantage of the calculation in the external field - we have to fix explicitly the gauge of $\mathrm{SU}(3)$ field. This problem could be resolved by dividing the four-potential $A_{\mu}^{a}$ into two parts:

$$
A_{\mu}^{a}=\left(A_{\mu}^{a}\right)_{e x t}+a_{\mu}^{a},
$$

where $\left(A_{\mu}^{a}\right)_{\text {ext }}$ is the vacuum field while $a_{\mu}^{a}$ denotes the hard gluon field. It is a matter of convenience to choose an additional term fixing the gauge of $a_{\mu}^{a}$ in the following form (so called background gauge):

$$
-\frac{1}{2}\left(D_{\mu}^{e x t} a_{\mu}^{a}\right)^{2}
$$

where $D_{\mu}^{e x t} a_{\nu}^{a}=\partial_{\mu} a_{\nu}^{a}+g_{s} f^{a b c}\left(A_{\mu}^{b}\right)_{e x t} a_{\nu}^{a}$. It is easy to see that the gauge invariance for $\left(A_{\mu}^{a}\right)_{\text {ext }}$ field is still preserved. The hard gluon propagator in the external field in this gauge takes the form [12]:

$$
\int i \exp \{i q x\} d^{4} x\left\langle T a_{\mu}^{a}(x) a_{\nu}^{b}(y)\right\rangle=\left(\delta^{a b} \frac{g_{\mu \nu}}{q^{2}}-2 i g_{s} f^{a c b} A_{\lambda}^{c}(y) \frac{q_{\lambda}}{q^{4}}+\frac{2 g_{s}}{q^{4}} f^{a c b} G_{\mu \nu}^{c}+\ldots\right) \exp \{i q x\},
$$

where we have omitted the subscript "ext". This form of propagator is very useful for our problem and it allows us to calculate CEDM covariantly without fixing the gauge of external field.

To reduce the number of diagrams the limit of large $N_{c}$ is used. In that limit combinations $t^{a} t^{a} t^{b} ; \quad$ if ${ }^{a b c} t^{a} t^{c} \simeq \frac{N}{2} t^{b}$ are much larger than $t^{a} t^{b} t^{a}=\frac{1}{2 N} t^{b}$. It means in particular that the external chromoelectric field can not be attached to the fermion line inside hard gluon loop as it shown at Fig.3. The accuracy of this approximation is presumably $\frac{1}{N_{c}^{2}}=1 / 9$ and it is within the errors connected with previous assumptions.

Let us first evaluate the contribution to this vertex from the operator $\mathcal{O}_{1} \sim \gamma_{\nu} D_{\mu} G_{\mu \nu}$ which is a usual "penguin". By virtue of the equation of motion

$$
D_{\mu} G_{\mu \nu}^{a}=-g_{s} \bar{q} \gamma_{\nu} t^{a} q
$$

and Fiertz transformation this operator generates a close fermion loop as it is shown in Fig. 4. The use of this equation is valid if the characteristic momenta inside this loop could be 
regarded smaller than $m_{h}\left(m_{g}\right)$ which is satisfied to logarithmic accuracy. Both topologies of W-boson attachment generates a nonvanishing contribution to CEDM of s-quark. It turns out, however, that to logarithmic accuracy there is a cancellation between these two types of diagrams and therefore penguins operators cannot contribute to the effect at the level of $\log ^{2}\left(m_{h}^{2} / m_{t}^{2}\right)$. It is possible to demonstrate that there is a nonvanishing contribution from these operators beyond this logarithmic approximation with a dependence of $m_{h}^{2} / m_{g}^{2}$ parameter. It requires tideous true multi-loop calculations which is beyond the scope of our purpose.

A similar cancellation between two topologies occurs when we substitute $\mathcal{O}_{2}$ operator and look for the effective vertex of W-boson with fermion. This operator, however, generates additional diagrams which should be taken into account as well. The first one represents a two loop mass operator inside W-boson loop (Fig. 5a); the second is the flavour changing mass operator inside gluonic loop (Fig. 5b). The crosses here indicate the chirality flips on the fermion line.

The calculation of inner mass operator depicted in Fig. 5a in use of operator $\mathcal{O}_{2}$ is simple. To logarithmic accuracy, it is given by

$$
M=\frac{c_{2}}{m_{w}^{2}} \frac{\alpha_{s} N_{c}}{4 \pi} m_{t} \log \left(\frac{m_{g}^{2}}{p^{2}}\right) g_{s}(G \sigma) \frac{1-\gamma_{5}}{2},
$$

where $p$ is the momentum flowing at the fermion line. It ranges between $m_{t}$ and the lightest mass of fourth generation quarks. The summation over flavours annihilates all other structures without the chirality flip. The only structure which does not vanish is the operator (39). Let us assume for the moment that $m_{h}^{2} \ll m_{g}^{2}$. Then this sum takes the form:

$$
\begin{array}{r}
{[(G \sigma), \hat{p}]\left(\frac{m_{t}^{2}}{p^{2}\left(p^{2}-m_{t}^{2}\right)} \log \left(\frac{m_{g}^{2}}{p^{2}}\right)-\frac{m_{h}^{2}}{p^{2}\left(p^{2}-m_{h}^{2}\right)} \log \left(\frac{m_{g}^{2}}{m_{h}^{2}}\right)+\right.} \\
\left.\frac{m_{h}^{2}}{\left(p^{2}-m_{t}^{2}\right)\left(p^{2}-m_{h}^{2}\right)} \log \left(\frac{m_{g}^{2}}{m_{h}^{2}}\right)\right) \simeq[(G \sigma), \hat{p}] \log \left(\frac{m_{h}^{2}}{p^{2}}\right),
\end{array}
$$

where we have neglected a further noncommutativity of momenta resulting from operators of dimension higher than that of CEDM. It is very natural that the logarithm is cut off at the lightest mass between $m_{h}$ and $m_{g}$. The last integration is also trivial and gives the CEDM operator of s-quark with a coefficient

$$
-\operatorname{Im}\left(V_{t s}^{*} V_{t b} V_{h b}^{*} V_{h s}\right) \frac{G_{F}}{\sqrt{2}} \frac{\alpha_{s} N_{c}}{4 \pi} \frac{1}{16 \pi^{2}} \frac{c_{2} m_{s} m_{t}^{2}}{m_{w}^{2}} \frac{1}{2 !} \log ^{2}\left(\frac{m_{h}^{2}\left(m_{g}^{2}\right)}{m_{t}^{2}}\right) .
$$

The last graph given by Fig. 5b contains flavour changing mass operator. Its value at the incoming momenta $k$, which is much larger than $m_{t}$, before the renormalization is given simply by:

$$
M(\hat{k})=\hat{k} f\left(k^{2}\right)\left(1-\gamma_{5}\right)=\frac{\alpha_{w}}{4 \pi} \frac{3}{4} \frac{m_{t}^{2}}{m_{w}^{2}} \hat{k} \log \left(\frac{\Lambda^{2}}{k^{2}}\right) \frac{1-\gamma_{5}}{2}
$$

where $\Lambda$ is the cut-off. The on-mass-shell renormalization prescription with respect to different masses to the left and to the right from the mass operator could be found in Refs. 
[3, 9. In our case this prescription looks trivial because both these masses are negligibly small in comparison with electroweak scale:

$$
M_{r}(\hat{k})=\hat{k}\left(f\left(k^{2}\right)-f\left(k^{2}=0\right)\right)\left(1-\gamma_{5}\right)=-\frac{\alpha_{w}}{4 \pi} \frac{3}{4} \frac{m_{t}^{2}}{m_{w}^{2}} \hat{k} \log \left(\frac{k^{2}}{m_{t}^{2}}\right) \frac{1-\gamma_{5}}{2} .
$$

The remaining integration over $k$ ranging between $m_{t}$ and $m_{h}\left(m_{g}\right)$ is equivalent to that performed several steps earlier, in (39). The corresponding contribution to CEDM of squark differs from (41) by the factor -3 . Combining these two numbers together and taking into account the value of the coefficient $c_{2}$, we obtain the final answer for the CEDM of s-quark for very heavy fourth generation, to double logarithmic accuracy and in the limit of large $N_{c}$ :

$$
\tilde{d}_{s}=-\operatorname{Im}\left(V_{t s}^{*} V_{t b} V_{h b}^{*} V_{h s}\right) \frac{G}{\sqrt{2}} m_{s} \frac{\alpha_{s} \alpha_{w}}{(4 \pi)^{4}} \frac{5 N_{c}}{12} \frac{m_{t}^{2}}{m_{w}^{2}} \frac{1}{2 !} \log ^{2}\left(\frac{m_{h}^{2}\left(m_{g}^{2}\right)}{m_{t}^{2}}\right) .
$$

Substitution of $N_{c}=3$ and $\frac{1}{2 !} \log ^{2}\left(\frac{m_{h}^{2}\left(m_{g}^{2}\right)}{m_{t}^{2}}\right) \sim 1$ to this formula yields the estimation of CEDM close to (22).

\section{Conclusions}

We have demonstrated a new interesting feature of the model with four generations of quarks incorporated into the same KM mechanism. The enhancement of neutron EDM in comparison with SM prediction comes from small distance effects which provide a regular factor of order $m_{t}^{2} / m_{c}^{2}$. The resulting value of EDM in this model, however, is just two orders of magnitude larger than corresponding SM value. The reason for that is in the numerical importance of large distance contribution in SM which is two orders of magnitude bigger than that coming from EDMs or CEDMs of quarks.

The estimation of relevant operators in the effective Lagrangian performed in this work allows one to consider the influence of the fourth generation on other low-energy CPviolating observables such as T-odd form-factors of heavy nuclei. This problem deserves special consideration.

\section{Acknowledgements}

We thank C. Burgess, V. Chernyak, G. Couture, V. Khatsimovsky, I. Khriplovich, A. Vainshtein and A. Yelkhovsky for helpful stimulating discussions. 


\section{Figures}

Fig. 1a

Fig. 2a

Fig. 3

Fig. 4
Fig. 1b

Fig. 2b

Fig. 5b 


\section{References}

[1] K.F. Smith et al., Phys. Lett. B234 (1990) 191; I.S. Altarev et al., Phys. Lett. B276 (1992) 242.

[2] C. Hamzaoui, A.I. Sanda, A. Soni, Phys. Rev. Lett. 63 (1989) 128.

[3] E.P. Shabalin, Yad.Fiz. 28 (1978) 151 (Sov. J. Nucl. Phys. 28 (1978) 75).

[4] I.B. Khriplovich, Yad.Fiz. 44 (1986) 1019 (Sov. J. Nucl. Phys. 44 (1986) 659).

[5] I.B. Khriplovich and A.R. Zhitnitsky. Phys. Lett. B109 (1982) 490.

[6] M.B. Gavela et al. Phys. Lett. B109 (1982) 215.

[7] X.-G. He, B.J.H. McKellar and S. Pakvasa. Peprint UH-511-666-89 (1989).

[8] S. Weinberg, Phys. Rev. Lett. 63 (1989) 2333.

[9] M.E. Pospelov, Phys. Lett. B328 (1994) 441.

[10] A. De Rujula, M.B. Gavela, O. Pene and F.J. Vegas, Nucl. Phys. B357 (1991) 277.

[11] V.M. Khatsimovsky, I.B. Khriplovich and A.R. Zhitnitsky, Z. Phys. C36 (1987) 455.

[12] V.A.Novikov, M.A. Shifman, A.I. Vainshtein and V.I.Zakharov, Fortschr. Phys. 32 (1984) 585. 
This figure "fig1-1.png" is available in "png" format from: http://arxiv.org/ps/hep-ph/9503468v1 